\title{
Attentional resources in dual-task performance
}

\author{
SOLEDAD BALLESTEROS \\ Universidad Nacional de Educación a Distancia, Madrid, Spain \\ and \\ DIONISIO MANGA and TERESA COELLO \\ Universidad Complutense, Madrid, Spain
}

\begin{abstract}
In this study, the time-sharing interference paradigm, which involves the concurrent performance of two activities, was used. Subjects performed unimanual tapping (a simple motor task) simultaneously with discrimination of pronounceable four-letter nonwords or sets of four spatially oriented lines (a cognitive task). In addition, two types of instructions, requiring global or local processing, were given to the subjects. Interference was larger with the right unimanual tapping (left hemisphere), and with the performance of the cognitive task during use of the local strategy. The results seem to accord with Kahneman's theory of limited attentional resources and with the notions of hemispheric specialization and functional cerebral space.
\end{abstract}

The time-sharing interference paradigm, initially developed by Kinsbourne and Cook (1971), involves the concurrent performance of two activities. It reveals the lateralization of verbal output control in normal subjects. The rationale for the vocal-manual interference method relies on the assumption that when subjects perform two unrelated tasks simultaneously, a situation involving interference is created. Subjects perform better when the two tasks are controlled by different cerebral hemispheres than when they are controlled by the same hemisphere. The time-sharing paradigm is based on the functional cerebral distance principle (Kinsbourne, 1982; Kinsbourne \& Hicks, 1978; Kinsbourne \& Hiscock, 1983). According to this principle, the greater the functional distance between two cerebral loci, the less likely or severe the contamination will be, and, therefore, the better able the operator will be to perform both tasks simultaneously with a relatively high level of efficiency.

The time-sharing paradigm has served to demonstrate vocal-manual interference in children and adults when there is competition between these two motor processes. It is supposed that competition for the same neural mechanisms of motor output is produced. The most common procedure has been the computation of the interference of speech during unimanual tapping. The interference is larger for the right hand than for the left. It is thought that this asymmetry occurs because both tasks compete for resources in the left hemisphere. Competition between a motor process and a cognitive process has also been shown, even though the separation between motor-motor and cognitive-motor is sometimes arbitrary

The authors thank Beryl McKenzie, La Trobe University, Australia, and Lynn A. Cooper, Columbia University, for helpful comments on an early version of this manuscript. Requests for reprints should be sent to Soledad Ballesteros, Department of Psychology, Universidad Nacional de Educación a Distancia, P.O. Box 50487, Madrid, Spain. (see Kinsbourne \& Hiscock, 1983). In addition, some tasks without obvious motor components have produced asymmetric interference in manual performance (Hellige \& Longstreth, 1981).

When two tasks are performed at the same time, there is some cost. The usual way to explain dual-task interference is to suppose that the two tasks make use of the same limited resources, and that when the demand exceeds the existing supply, the processing of the two tasks involves competition so that interference occurs (Navon $\&$ Miller, 1987). The research on processing capacity in terms of attentional resources suggests that if the degree of similarity between the concurrent tasks is held constant, the capacity to perform both tasks diminishes when the difficulty of one or both of them increases. It is known that difficult tasks require a larger cortical mass for the propagation of activation than easy tasks do, and that difficult tasks have larger attentional demands; easier or more automatized tasks need less cortical mass for propagation of activation.

In this study, unimanual tapping served as a simple motor task, while the cognitive task consisted of the discrimination of pronounceable nonwords and sets of four spatially oriented lines. The nonwords differed in only one of the four letters, and the sets of lines differed only in the orientation of one of the four lines. In addition, two types of instructions were given to the subjects, requiring either global or local processing. Local processing requires generally larger attentional demands (Neisser, 1967), so it was hypothesized that there would be less interference in the global condition. The global precedence literature (Broadbent, 1977; Miller, 1981; Navon, 1977) suggests that perceptual processing proceeds from global structuring toward analysis of more local details, with perceptual priority for global forms. There are, however, several boundary conditions for this phenomenon. Several variables affect global-local dominance, such as stimu- 
lus size (Kinchla \& Wolfe, 1979), sparsity of local elements (Martin, 1979), number and relative size of local elements (Kimchi \& Palmer, 1985), and integrality or separability of the dimensions involved (Kimchi, 1988). For a recent review, see Lasaga (1989).

\section{METHOD}

\section{Subjects}

The subjects were 16 female graduate students aged from 20 to 23 years. They were selected from 140 students who participated in an experiment on the discrimination of nonwords and lines; 8 were very fast and the other 8 were very slow in line discrimination. All were righthanded, and without familial sinistrality. Their manual laterality quotients ranged from 65 to 100 , according to Oldfield's inventory (Oldfield, 1971).

\section{Stimuli and Apparatus}

The stimuli, printed in booklets, were four-letter Spanish nonwords and sets of four straight lines of different orientation (horizontal, verti$\mathrm{cal}$, and the two diagonals). One nonword or a set of lines appeared as the reference stimulus in the left side of the booklet, and four search stimuli appeared on the right side (two were the same as, and two different from, the reference stimulus). The difference was a letter or a line orientation.

The tapping apparatus had two metal plates, one on the right and one on the left, which were connected to a counter; a stylus was provided for tapping on the plates. The counter was programmed to register 20-sec periods.

\section{Procedure}

One hundred and forty subjects took part in a group task, which consisted of crossing out the nonwords and sets of lines that differed from the reference stimuli. A sound each $20 \mathrm{sec}$ marked their crossing-out rhythm. The task lasted $2 \mathrm{~min}$. The cognitive-task baseline was thus established, and the fast and slow subjects were selected from this larger sample.

To obtain the tapping-task baselines for each subject, the subject was asked to tap as quickly as possible, first with the right hand and then with the left. Once both baselines were obtained, each subject participated in the dual-task condition. The experiment began with right-handed tapping while the left hand (the free hand) was pointed to the "different" elements of the visual discrimination task. After $20 \mathrm{sec}$, the tasks for the hands were reversed. There were two experimental conditions, depending on the instructions. In the global condition, the subjects had to point to the "different" stimulus sets. In the local condition, the subjects had to indicate with a pencil the components that differed in the "different" sets. The subjects were asked to point, not to mark, in order to avoid possible motor implications of the cognitive task. Half of the subjects in each group (fast and slow) began with the global condition, and the other half with the local condition. Within every 20 -sec period, there were as many trials as the subjects could complete.

\section{RESULTS}

Following previous procedures (e.g., those of Hughes \& Sussman, 1983, and White \& Kinsbourne, 1980), tapping rates were transformed into percentage-change scores using the following formula:

$$
\left[\left(\text { taps }_{\text {control }}-\text { taps }_{\text {concurrent task }}\right) /\left(\text { taps }_{\text {control }}\right)\right] \times 100 \text {. }
$$

Cognitive scores were also transformed into percentagechange scores by means of the same formula.

A mixed factorial design was used, with 2 (group: fast or slow) $\times 2$ (hand: left or right) $\times 2$ (material: nonwords or lines) $\times 2$ (strategy: global or local) $\times 2$ (task: tapping or discrimination) as factors. Group was a

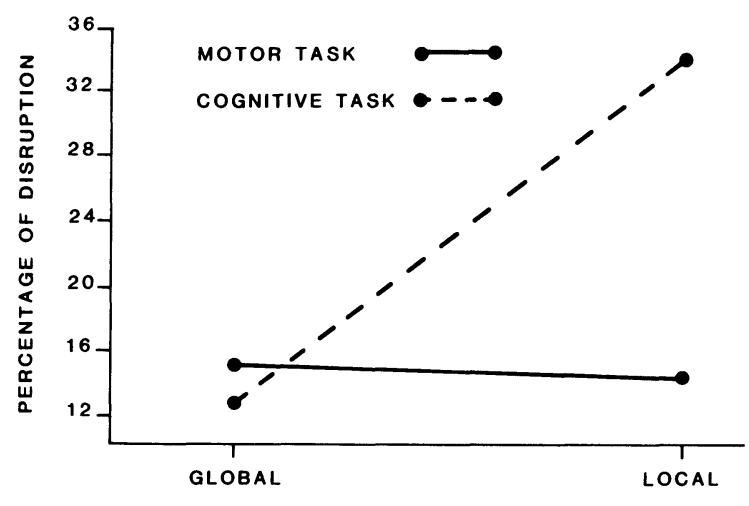

Figure 1. The interaction between type of strategy (global and local) and task.

between-subjects factor. Analysis revealed a significant main effect for group $[F(1,14)=6.64, p<.05]$, hand $[F(1,14)=40.2, p<.001]$, material $[F(1,14)=77.03$, $p<.001]$, and strategy $[F(1,14)=23.22, p<.001]$. The following two-way interactions were also significant: material $\times$ group $[F(1,14)=19.75, p<.001]$; task $\times$ group $[F(1,14)=4.81, p<.05]$; hand $\times$ task $[F(1,14)$ $=14.17, p<.01]$; material $\times$ task $[F(1,14)=86.26$, $p<.01]$; strategy $\times$ task $[F(1,14)=54.77, p<.001]$. Finally, the three-way interaction of material, task, and group was also significant $[F(1,14)=22.49, p<.001]$.

The strategy main effect showed that the global-strategy interference was smaller than the local one $(14.31 \%$ vs. 24.54\%).

Figure 1 shows the strategy $\times$ task interaction; analysis of the global strategy with the Newman-Keuls test showed that there was no significant difference between tapping and discrimination, but that with the local strategy the difference was significant, the interference being larger in the cognitive task.

The hand main effect showed that the right-hand interference was always significantly larger than the left-hand interference $(24.38 \%$ vs. $14.48 \%)$. This result indicates an asymmetry between hemispheres, with larger interference when the left hemisphere was involved in the motor task simultaneously performed in conjunction with the cognitive task. Figure 2 shows the interaction between hand and task. The Newman-Keuls test showed that the

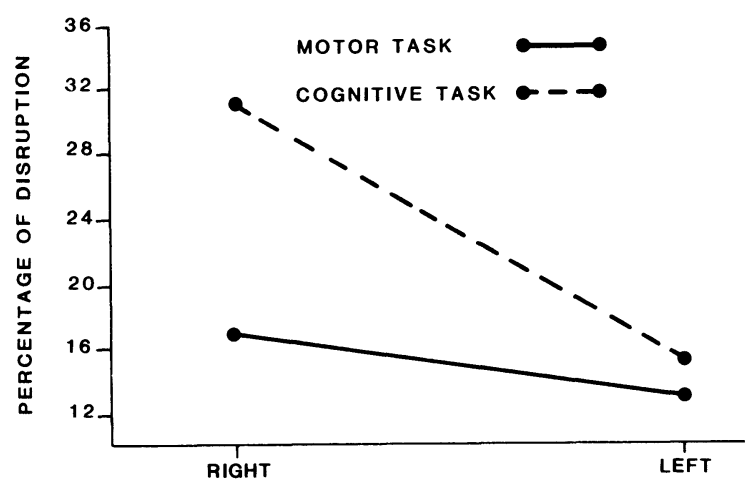

Figure 2. The interaction between hand (right or left) and task. 
difference between motor and cognitive tasks was significant when performance involved the right hand $(17.42 \%$ vs. $31.34 \%$ ) but not the left.

The fast group showed larger interference than the slow group $(24.42 \%$ vs. $14.43 \%)$. The group $\times$ task interaction showed that whereas the slow group did not exhibit mean significant differences between tasks, for the fast group there was a larger difference in the cognitive task than in the motor task (34.48\% vs. $14.37 \%)$. The group $\times$ material interaction showed that the two groups differed only in line but not in word discrimination, with the interference for the fast group being larger $(20.29 \%$ vs. $0.96 \%$ ), because the selected groups were the two extremes in the line task. The three-way group $\times$ task $\times$ material interaction showed that the fast-group difference between the motor task and the cognitive task occurred only for nonwords, whereas the slow group showed this difference only for lines.

\section{DISCUSSION}

Our results suggest that local processing demands more attentional resources, because the reduction in task performance was larger when the subjects used the local strategy than when they used the global one. It is interesting that the interference was larger in the cognitive than in the motor task. The motor-task interference was not modified by the change in strategy. In our data, there was no significant difference between motor and cognitive interference, and only the cognitive interference increased with the larger amounts of attentional demands in the local strategy. Following the functional cerebral distance principle, the motor task is more easy and automatic, with minimum cortical propagation after a certain amount of practice. The cognitive task with the local strategy requires more attentional demands and cortical propagation than it does with the global strategy. In the cross-talk between the respective regions, an increment is produced in the side in which attentional demands are also increased.

Right-hand interference is larger than left-hand interference. This asymmetry seems to show that the most important competition between motor and cognitive processes takes place in the left cerebral hemisphere. There is no difference between the motor and cognitive reduction in left-hand tapping (right hemisphere), but there is a significant difference in right-hand tapping (left hemisphere). Again, interference increased in the cognitive task when tapping was performed with the right hand. We are faced with a well-known finding in the cerebral hemispheric specialization literature (Bradshaw \& Nettleton, 1981); the type of material and the mode of processing in the cognitive task were especially analytical, which necessitated more left-hemisphere involvement.

In summary, our data seem to accord with Kahneman's (1973) notion of limited attentional resources, appearing as an interference between concurrent tasks that involve competition for central resources. Even though Hirst (1986) has stated that several studies provide evidence against the idea that dual-task decrements result from resource competition and some experiments have shown that resources must expand with practice, task interference is a robust phenomenon that cannot be eliminated. There was interference when the tapping was performed with the right hand and when it was performed with the left; both hemispheres seem to have been implicated. But the interference was larger in the left hemisphere (right unimanual tapping), during performance on the cognitive task and use of the local strategy. This interference fits with the notions of hemispheric specialization and the principle of cerebral functional space. Finally, the differences between groups, with less interference shown in the slow group, suggest that there are subject parameters like those put forth by Navon and Gopher (1979). In any case, it is necessary to distinguish between dual-task in- terference effects and the asymmetry of this effect (see Kamhi \& Masterson, 1986). Some tasks are associated with small asymmetry and large interference, and others with the opposite. Friedman and Polson (1981) recommend using tasks that utilize resources from one or the other hemisphere, because otherwise the interpretation of the results can be difficult. We did not find significant interaction between hand and type of material, which could mean that the two types of stimulus materials (nonwords and oriented lines) were processed by the same hemisphere. Furthermore, it would be necessary to take into account all the methodological issues related to the dual-task paradigm (Green \& Vaid, 1986), should one want to reduce the risk of error in the interpretation of the results.

\section{REFERENCES}

Bradshaw, J. L., \& Nettleton, N. C. (1981). The nature of hemispheric specialization in man. Behavioral \& Brain Sciences, 4, 109-118.

BroAdBEnT, D. (1977). The hidden preattentive processes. American Psychologist, 32, 109-118.

Friedman, A., \& Polson, M. C. (1981). Hemispheres as independent resource systems: Limited-capacity processing and cerebral specialization. Journal of Experimental Psychology: Human Perception \& Performance, 7, 1031-1058.

GreEN, A., \& VAID, J. (1986). Methodological issues in the use of the concurrent activities paradigm. Brain \& Cognition, 5, 465-476.

Hellige, J. B., \& LongSTRETH, L. E. (1981). Effects of concurrent hemisphere-specific activity on unimanual tapping rate. Neuropsychologia, 9, 1-10.

Hirst, W. (1986). The psychology of attention. In J. E. LeDoux \& W. Hirst (Eds.), Mind and brain (pp. 105-141). New York: Cambridge University Press.

Hughes, M., \& SuSSMAN, H. M. (1983). An assessment of cerebral dominance in language-disordered children via a time-sharing paradigm. Brain \& Language, 19, 48-64.

Kahneman, D. (1973). Attention and effort. Englewood Cliffs, NJ: Prentice-Hall.

KAMHI, A. G., \& MASTERSON, J. J. (1986). The reliability of the timesharing paradigm. Brain \& Language, 29, 324-341.

Kıмсні, R. (1988). Selective attention to global and local levels in the comparison of hierarchical patterns. Perception \& Psychophysics, 43, 189-198.

KImchI, R., \& PALmer, S. E. (1985). Separability and integrality of global and local levels of hierarchical patterns. Journal of Experimental Psychology: Human Perception \& Performance, 11, 673-688.

KINCHLA, R. A., \& WolfE, J. M. (1979). The order of visual processing: "Top-down," "bottom-up," or "middle-out." Perception \& Psychophysics, 25, 225-231.

KINSBOURNE, M. (1982). Hemispheric specialization and the growth of human understanding. American Psychologist, 37, 411-420.

KinsBourne, M., \& CoOK, J. (1971). Generalized and lateralized effect of concurrent verbalization on a unimanual skill. Quarterly Journal of Experimental Psychology, 23, 341-345.

KinSBouRNe, M., \& Hicks, R. E. (1978). Functional cerebral space: A model for overflow, transfer, and interference effects in human performance. In J. Requin (Ed.), Attention and performance VII. Hillsdale, NJ: Erlbaum.

KinSBourne, M., \& Hiscock, M. (1983). Asymmetries of dual-task performance. In J. B. Hellige (Ed.), Cerebral hemisphere asymmetry (pp. 255-334). New York: Praeger.

LASAGA, I. M. (1989). Gestalts and their components: Nature of the information precedence. In B. E. Shepp \& S. Ballesteros (Eds.), Object perception: Structure and process (pp. 165-202). Hillsdale, NJ: Erlbaum.

MARTIN, M. (1979). Local and global processing: The role of sparsity. Memory \& Cognition, 7, 476-484.

MiLLER, J. (1981). Global precedence in attention and decision. Journal of Experimental Psychology: Human Perception \& Performance, 7, 1161-1174. 
Navon, D. (1977). Forest before trees: The precedence of the global features in visual perception. Cognitive Psychology, 9, 353-383.

Navon, D., \& Gopher, D. (1979). On the economy of the humanprocessing system. Psychological Review, 86, 214-255.

NAvon, D., \& Miller, J. (1987). Role of outcome confict in dual-task interference. Journal of Experimental Psychology: Human Perception \& Performance, 13, 435-448.

NeISSER, U. (1967). Cognitive psychology. New York: AppletonCentury-Crofts.
OLDFIELD, R. C. (1971). The assessment and analysis of handedness: The Edinburgh Inventory. Neuropsychologia, 9, 97-113.

White, N., \& KinsBourne, M. (1980). Does speech output control lateralize over time? Evidence from verbal-manual time-sharing tasks. Brain \& Language, 10, 215-223.

(Manuscript received January 3, 1989.)

\title{
Announcement
}

\author{
30th Annual Meeting of the Psychonomic Society \\ Atlanta, Georgia \\ November 17-19, 1989
}

The 30th Annual Meeting of the Psychonomic Society will be held in Atlanta, Georgia, November 1719, 1989. The meetings will begin Friday morning and continue until Sunday at noon. The headquarters hotel will be the Hyatt Regency.

The program and hotel reservation cards will be mailed to members and associates in September. A copy of the program will be published in the November issue of the Bulletin of the Psychonomic Society.

For further information, please contact the secretary-treasurer of the Society: Michael E. Rashotte, Department of Psychology, Florida State University, Tallahassee, FL 32306-1051 (Telephone: 904-644-2040; BITNET: Rashot@FSU). 